\title{
Kraniche im Spreewald und Biber in den Elbtalauen
}

\author{
Während vor allem Sportsponsoring, jedoch auch Sozial- und Kultursponsoring \\ von den Unternehmen häufig zur eigenen Imageförderung genutzt werden, sind \\ Aktivitäten des Öko-Sponsoring nur selten zu finden. Der nachfolgende Beitrag \\ klärt über Hintergründe auf, diskutiert Chancen und Probleme des Umweltspon- \\ sorings von Unternehmen und fasst die Erfahrungen der beteiligten Institutio- \\ nen zusammen.
}

\section{I} Von Katarina Jokovic, Oliver Krempig, Dunja Saleh-Zaki und Martin Wenke gazins (9/99) wurden die Ergebnisse einer Studie des Hamburger Umweltinstituts zur ÖkoPerformance der größten Chemiekonzerne weltweit veröffentlicht. Dabei spielten neben der verfolgten Umweltpolitik, den Umweltstandards, dem Umweltmanagement und anderen insgesamt zehn Kriterien auch die Unterstuitzung von Naturschützern und Umweltinitiativen eine Rolle. Mit dem insgesamt im Ranking auf dem dritten Platz liegenden Unternehmen Johnson\&Johnson (USA) wurde nur einem einzigen der ausgewiesenen 50 Chemiefirmen diesbezüglich ein ,sehr gut“ attestiert. Unter den ersten Zehn wurden aber immerhin sechs Unternehmen hinsichtlich ihres externen Öko-Engagements mit ,gut“ bewertet. Firmen am unteren Ende der Rangskala wiesen demgegenüber durchweg eine nur ausreichende oder mangelhafte Beurteilung auf. Somit scheinen gute Öko-Performance und hohes externes Umweltengagement einander zu bedingen.

Für die Mehrheit der Unternehmen spielt diese Art der Unterstützung etwa im Rahmen eines Öko-Sponsorings jedoch keine Rolle. Dies gilt in der Regel auch für diejenigen Unternehmen, die mit der Beteiligung an zertifizierten Umweltmanagementsystemen eine besondere umweltbezogene Sensibilisierung erkennen lassen.

Beim Öko-Sponsoring stellen Unternehmen Geld, Sachmittel oder Dienstleistungen zur Erfüllung ökologischer Aufgaben zur Verfügung. Im Unterschied zum Spendenwesen verfolgen die Unternehmen die Intention einer konkreten Förderung von Projekten und/oder Institutionen, die den Schutz der Umwelt zum Ziel haben. Umweltorganisationen sorgen für die sachgerechte und ziel- gerichtete Verwendung dieser Mittel. Nahezu alle großen Umweltverbände wie NABU, BUND, oder die Deutsche Umwelthilfe (DUH) akzeptieren und praktizieren Umweltsponsoring als Form der Mittelbeschaffung, allerdings mit erheblichen Unterschieden bei den Kriterien, die an die Partner aus der Wirtschaft gelegt werden. Dabei macht sich zunehmend ein verändertes Rollenverständnis beider Gruppen bemerkbar: War früher eher Konfrontation zwischen Umweltverbänden und Unternehmen die Regel, werden immer häufiger die Vorteile einer Kooperation erkannt: Vor allem die Unternehmen erkennen, dass neben der beabsichtigten Imageverbesserung erhebliche Fortschritte beim betrieblichen Umweltschutz erzielt werden können, wenn mit den Experten der Umweltverbände eine entsprechende Beratungskooperation vereinbart wird (1).

\section{- Kriterien der Partnerwahl}

Öko-Sponsoring kann als Förderung einer Organisation, eines Gesamt- oder eines Teilprojektes erfolgen. Ein besonders wichtiger Aspekt ist die Auswahl der jeweiligen Partner. Sowohl für die Unternehmen als auch für die Umweltverbände erweist es sich als sinnvoll, jeweils die Kriterien und Erwartungen des potenziellen Partners zu überprüfen, die für eine zukünftige Kooperation von maßgeblicher Bedeutung sind. Da beim Sponsoring der gegenseitige Austausch von Leistungen im Vordergrund steht, ist es für die Umweltverbände wichtig, genau über die Firmenphilosophie des in Betracht kommenden Sponsors informiert zu sein. Neben den in Abbildung 1 genannten Einzelkriterien verlangt zum Beispiel die DUH ,lediglich“ die Grundbedingungen einer Umweltmarktfuihrerschaft, die Gleichrangigkeit von ökonomischen, ökologischen und sozialen Zielen sowie das Bekenntnis zur nachhaltigen Entwicklung und der Agenda 21. Die Unternehmen ihrerseits sollten sich auf die Professionalität der Organisation verlassen können, damit Glaubwürdigkeit und Image nicht gefährdet werden.

\section{Akzeptanzprobleme der Firmen}

Das Thema Umwelt muss als wesentlich sensibler bezüglich der Wahrnehmung durch die Öffentlichkeit angesehen werden und ist deutlich stärkeren Aufmerksamkeitsschwankungen unterworfen als die Bereiche Sport, Kultur oder Soziales. Des weiteren stellt Umweltsponsoring sehr hohe Anforderungen an die Unternehmen; zum einen durch die Anforderungsprofile der Umweltverbände, zum anderen durch die auch für die öffentliche Akzeptanz unabdingbare - Notwendigkeit der Übereinstimmung des Umweltverhaltens des Unternehmens mit den Zielen des unterstïtzten Umweltverbandes oder Projektes.

\section{- Öko-Sponsoring in der Praxis}

Im Rahmen eines Projektseminars am Fachbereich Wirtschaft der Fachhochschule Niederrhein wurden Umweltverbände und Unternehmen über ihre Erfahrungen befragt. Nach einer Einschätzung von Jörg Dürr-Pucher von der Deutschen Umwelthilfe lag die Hoch-Zeit des Umweltsponsoring in den Jahren 1995/96. Danach investier-

\section{Abbildung 1: Kriterien zur Beurteilung der Sponsoring-Partner}

... aus der Wirtschaft

- keine Gesundheitsgefährdung der Produkte und Leistungen

hoher Erfüllungsgrad des erwarteten Produktnutzens

- geringer Ressourcenverbrauch durch Mehrweg, Langlebigkeit und Recyclingfähigkeit der Produkte

Fördersumme und Verträglichkeit mit bestehenden Sponsorbeziehungen

Fremdimage des Unternehmens

... aus dem Bereich der Umweltverbände

- Professionalität und Effektivität der Arbeit

- Existenz konkreter Projektkonzepte

- Akzeptanz des Unternehmens als gleichberechtigter Partner

Auftreten in der Öffentlichkeit

Quelle: in Anlehnung an Rahmel, D./Zillesssen, R.: Umweltsponsoring. Gabler 1991, S. 63 
Abbildung 2: Öko-Sponsoring-Projekte - ausgewählte Beispiele

\begin{tabular}{l|l|l} 
Projekt & Umweltverband & Unternehmen \\
\hline $\begin{array}{l}\text { Hilfe für den Weißstorch,Artenschutzkoffer, } \\
\text { Umweltbuch, Elbtalauen }\end{array}$ & WWF, NABU & OTTO-Versand \\
Blauer Planet - Für aktiven Umweltschutz & Deutscher Naturschutzring & Gerolsteiner Brunnen \\
Neue Nationalparke in Ostdeutschland & pro Natur & WELLA \\
Elbtalauen & DUH & KYOCERA \\
$\begin{array}{l}\text { Erhalt der Natur des Truppenübungsplatzes } \\
\text { "Liberose“, Blumberger Mühle }\end{array}$ & NABU & Tengelmann \\
Ökologischer Teeanbau & NABU, TransFair & Der Teeladen \\
Schutz des Wattenmeeres & WWF & Holsten Brauerei \\
Müritz Nationalpark & WWF & Canon
\end{tabular}

ten die Firmen aufgrund des Booms der Fußballbundesliga und der Formel 1 wieder deutlich mehr in das Sport-Sponsoring, zu Lasten des Umweltsponsoring. Zur Kontaktaufnahme mit Unternehmen werden von der DUH neben schriftlichen und telefonischen Ansprachen Pressereisen und Meetings veranstaltet, auf denen über neue Projekte informiert wird. Insgesamt ist nach Einschätzung von DUH und NABU das Interesse an Umweltsponsoring-Aktivitäten rückläufig. Wenn sich allerdings Unternehmen zu einer Zusammenarbeit entschlossen haben, gehen sie in der Regel längerfristige Partnerschaften ein. Aus den Informationsmaterialien des BUND geht hervor, dass die Chancen des Umweltsponsoring neben der Erschließung von Finanzierungsquellen vor allem in der Beeinflussung der Unternehmenspolitik gesehen werden.

Darüber hinaus setzt die Organisation ihre Autorität bei den Verbrauchern dafür ein, umweltverträgliche Produkte auf dem Markt durchzusetzen. Als besondere Risiken werden vor allem ein mögliches umweltbezogenes Fehlverhalten der Partner und der dann entstehende Imageverlust für die Organisation gesehen. Auch die Gefahr, dass die Kooperation mit Unternehmen als Käuflichkeit des Umweltschutz interpretiert werden könnte, wird gesehen. Die internationale Umweltorganisation WWF nennt als Ziele der Kooperation mit Unternehmen die Förderung umweltverträglichen Wirtschaftens, die Bewusstseinsbildung der Unternehmensverantwortlichen und Mitarbeiter sowie die Finanzierung der Arbeiten des Verbandes. Neben dem projektbezogenen Sponsoring erzielt der WWF auch Einnahmen über die Möglichkeit der Verwendung des Umwelt-Logos durch die Partner auf Lizenzbasis.

Mit Blick auf die beteiligten Unternehmen ist es nicht verwunderlich, dass sich vor allem solche Unternehmen über des Umweltsponsoring dem
Schutz der natürlichen Lebensgrundlagen widmen, die diese Grundlagen als Produktionsfaktoren nutzen (vergleiche Abbildung 2). Dies gilt vor allem für Unternehmen der Lebensmittelindustrie wie die Holsten-Brauerei oder aber die Tengelmann Warenhauskette, den Teeladen, den Nestlé-Konzern oder das Unternehmen HIPP. Andere Unternehmen stellen häufig mit Hilfe ihrer ausgeprägten Umweltphilosophie sowie der Umweltfreundlichkeit von Gütern und Leistungen bzw. der Produktionsprozesse eine Verbindung zu den gesponserten Verbänden und Projekten her. Ein gutes Beispiel dafür, wie sich Umweltsponsoring quasi automatisch aus der vollständigen Integration des Umweltgedankens ergibt, stellt der japanische KYOCERA-Konzern dar, der sich durch die Zertifizierung aller Tochterunternehmen nach ISO 9.000 und 14.000 sowie das Angebot des ersten mit dem Umweltzeichen „Blauer Engel“ ausgezeichneten PC-Druckers hervorhebt. In Zusammenarbeit mit der DUH wird seit 1993 das Umweltprojekt „Schutz der Elbtalauen“ gefördert. Und mit den Partnern Gruner+Jahr und StorckStiftung unterstiutzt man die dazugehörige Wanderausstellung.

Wie viele andere der befragten Unternehmen so bestätigte auch Detlef Herb, Umweltbeauftragter von KYOCERA, dass die Ziele des Sponsoring wie etwa die Verbesserung der Kommunikation mit Händlern und Endkunden bisher nur unzureichend erreicht werden konnten. Zwar sei eine Imageverbesserung nach außen sowie eine verstärkte Sensibilisierung der MitarbeiterInnen nach innen feststellbar. Inwieweit sich dies in einer verbesserten Marktposition des Unternehmens niedergeschlagen habe, sei jedoch nicht abschließend zu beurteilen. Lediglich einige Großkunden hätten positiv auf die Umwelt-Aktivitäten von KYOCERA reagiert.
Unternehmen, die auf der Suche nach Sponsorship-Nehmern sind, können auch das Internet als Informationsquelle nutzen. Neben den einzelnen Internetseiten der Umweltverbände kann das Angebot der Europäischen Sponsoring-Börse ESB in Anspruch genommen werden (2). Nur wenige Unternehmen werben aktiv mit Sponsoring-Aktivitäten auf ihren Homepages.

\section{Ausblick}

Viele Umweltschutzaktivitäten der Unternehmen, die zunächst einmal nur mit Kosten verbunden sind und außer einer Imageverbesserung keine nennenswerten ,returns on investment“ erzielen, verzeichnen derzeit eine Flaute. Dies gilt auch für das Öko-Sponsoring. In Umwelterklärungen einerseits und entsprechenden Veröffentlichungen und Presseterminen andererseits verfahren die Unternehmen zwar nach dem Motto ,tue Gutes für die Umwelt und rede darüber“. Was nutzt dies allerdings, wenn die Zielgruppe, vor allem also Kunden und Endverbraucher, nicht hinhören bzw. den Informationen kaum Relevanz für die Kaufentscheidung einräumen? Dies könnte zur Konsumentenschelte führen, muss aber vor allem wohl Konsequenzen für die Kommunikationsstrategie der Unternehmen nach sich ziehen. Denn viele Unternehmen reden zu leise über ihre guten Taten. Hier scheint ein weithin unbestelltes Feld für Marketingstrategen vorzuliegen, dass schnellstens bearbeitet werden sollte.

\section{Anmerkungen}

(1) Vgl. z.B. die entsprechenden Ausführungen der DUH im jüngsten Umweltbericht des DaimlerChrysler-Konzerns, DaimlerChrysler (Hrsg.): Umweltbericht '99. Daten und Fakten, S. 39.

(2) Im Internet unter www.esb-online.com. Internet-Adressen der Umweltverbände: www.bund.net, www.nabu.de, www.duh.de, www.wwf.de

\section{Die Autorlnnen}

Katarina Jokovic, Oliver Krempig und Dunja SalehZaki sind Studierende am Fachbereich Wirtschaft der Fachhochschule Niederrhein (FHN), Prof. Dr. Martin Wenke ist dort für das Fachgebiet Ökonomie, Ökologie und Ethos verantwortlich.

Kontakt: FHN, Fachbereich Wirtschaft, Webschulstraße 41-43, 41065 Mönchengladbach, Tel. 02161/ 186-817, Fax -880,

E-mail: martin.wenke@fh-niederrhein.de 
(c) 20I0 Authors; licensee IÖW and oekom verlag. This is an article distributed under the terms of the Creative Commons Attribution Non-Commercial No Derivates License (http://creativecommons.org/licenses/by-nc-nd/3.o/), which permits unrestricted use, distribution, and reproduction in any medium, provided the original work is properly cited. 